\title{
EDITORIAL VOLUMEN 9 NÚMERO 1
}

\section{EDITORIAL VOLUME 9 ISSUE 1}

Se ha consolidado un nuevo número de la Revista de Psicología y Ciencias del Comportamiento de la U. A. C. J. S. y con ello, también, se sigue concretando la posibilidad de contribuir a la difusión del conocimiento científico en materia de comportamiento humano. En esta ocasión, los artículos que se presentan son en buena medida -según creemos-, una muestra de la diversidad de objetos de estudio, así como de perspectivas teóricas y metodológicas que caracterizan actualmente a las ciencias del comportamiento.

En primera instancia, en el artículo titulado "Estudio sobre el posicionamiento y la validación del artista en el ámbito cultural de Nuevo León, México" se examinan las condiciones sociales que determinan al artista como actor cultural, todo ello analizado desde un marco teórico propio de la Sociología del Arte y a través de una metodología cualitativa.

Desde abordajes metodológicos no experimentales y cuantitativos se nos presentan tres trabajos:

En "Prevalencia de violencia en el noviazgo en una muestra de varones adolescentes mexicanos", se analiza la frecuencia en que los varones de una muestra ejercen y sufren violencia en sus relaciones de noviazgo; haciendo uso de estadística inferencial correlacional los autores estudian también las posibles asociaciones entre variables como la duración de la relación y la violencia perpetrada dentro de la misma. Interesados por las bases fisiológicas del comportamiento humano, los autores del trabajo "Hostilidad, enojo y agresión: Diferencias fisiológicas en una muestra de mujeres universitarias del norte de México" se dan a la tarea de analizar las diferencias ya mencionadas, haciendo uso de métodos no experimentales y análisis estadísticos inferenciales. Relacionado al ámbito educativo, el artículo "Autoconcepto y apoyo social en estudiantes de bachillerato. Un estudio predictivo" expone los hallazgos que los autores obtuvieron al analizar las distintas dimensiones del apoyo social y el papel de éstas en la predicción del autoconcepto, todo ello haciendo uso de un modelo de regresión lineal múltiple.

Dentro del campo de los desarrollos psicométricos, el artículo "Evidencias de validez y confiabilidad de la Escala de Procrastinación Académica en una población estudiantil mexicana" nos presenta los resultados de la evaluación de las 
propiedades psicométricas de dicha escala, en una muestra de estudiantes de bachillerato, haciendo uso de herramientas estadístico-matemáticas como el análisis factorial exploratorio y confirmatorio.

Finalmente, por medio de un extenso análisis teórico el autor del artículo "La caída de los referentes en el mundo contemporáneo: análisis del concepto unario en Dany Robert-Dufour" estudia el concepto de lo unario, como forma de locura, y su relación con la caída de los referentes tradicionales (Dios, el Estado y la Razón) que durante largo tiempo han organizado la vida subjetiva de los individuos.

Esperamos que esta breve descripción de los trabajos que conforman el número en cuestión resulte sugestiva para los lectores que se dispongan ahondar en él, así como para los futuros autores cuyo deseo sea contribuir en nuestra revista, que por definición es abierta a todos los esfuerzos científicos y reflexivos emprendidos desde la rica paleta de métodos y enfoques teóricos que se gestan en el área del comportamiento humano.

El Comité Editorial. 